\title{
Institucionalización del predominio privado en la educación superior colombiana. Prefiguraciones del campo de políticas
}

INSTITUTIONALIZATION OF PREDOMINANCE PRIVATE OF COLOMBIAN HIGHER EDUCATION. PREFIGURATIONS OF POLICIE'S FIELD

I INSTITUCIONALIZACIÓN DO PREDOMINIO PRIVADO NA EDUCAÇÃO SUPERIOR COLOMBIANA. PREFIGURACIONES DO CAMPO DE POLÍTICAS

René Guevara Ramírez */ rguevara@pedagogica.edu.co

\begin{abstract}
Resumen
El artículo suministra claves comprensivas sobre la institucionalización del predominio privado en la oferta institucional y la alternancia sectorial en la matrícula de la educación superior colombiana, asumiendo como perspectiva analítica la progresiva configuración de su campo de políticas.
\end{abstract}

\section{Palabras clave}

Educación superior, universidades públicas, políticas universitarias, campo de políticas.

\section{Key words}

Higher education, public universities, university policies, policy field.

The article provides comprehensive keys on the institutionalization of the predo-
minance of private institutional supply and the sectoral alternation in the tuition of higher education in Colombia, assuming as analytical perspective to progressively develop their policy field.

\section{Resumo}

O artigo prevê chaves compreensivo sobre a institucionalização da predominância de privada na oferta institucional e sectorial alternância na matricula do ensino superior na Colômbia, assumindo como perspectiva analítica para desenvolver progressivamente o seu campo político.

\section{Palavras-chave}

Ensino superior, universidades públicas, políticas universitárias, campo da política.

\footnotetext{
* Profesor asistente de la Universidad Pedagógica Nacional, Colombia. Doctor en Ciencias Sociales, Universidad Autónoma Metropolitana, Xochimilco, México.
}

Fecha de recepción: 30 de marzo de 2014 / Fecha de aprobación: mayo 23 de 2014 


\section{Introducción}

La investigación de la cual se empieza a dar cuenta, publicando los primeros resultados parciales aquí contenidos 1 , estuvo orientada al establecimiento de condiciones de posibilidad configurativas del "Sistema" de educación superior colombiano. La pregunta de indagación tomada como centro de atención para la redacción del presente artículo es la siguiente: ¿Cómo se configura la posición ocupada por las universidades públicas dentro del espacio institucional de la educación superior colombiana?

Dicha pregunta remitiría, inicialmente, a la reconstrucción estadística de la historia constitutiva del referido "Sistema", implicando diferenciar momentos analíticos. Se observará, por tanto, la configuración de dicha estructura en su objetividad ${ }^{2}$, relacionalmente, contribuyendo a desnaturalizar significaciones subyacentes a la oferta institucional, resituando su composición sectorial (público-privado) en tanto que posiciones y posicionamientos institucionales, consecuentes con el devenir constitutivo de sus relaciones dentro del Estado colombiano.

Se aspira a que las entregas parciales de la investigación comentada sean observadas en la dimensión de una progresiva construcción, demandando consecuentes y paulatinas acumulaciones y distanciando la producción investigativa de alguna segmentación mecánica. En esta ocasión, será realizada la objetivación anunciada con miras a precisar el momento en que se instituiría el predominio del sector privado en la oferta institucional, y la alternancia sectorial en la matrícula de la educación superior colombiana, constituyendo un momento de la perspectiva de investigación asumida (Teoría de la práctica3).

1 Investigación realizada con financiación del CIUP-UPN, código DPG-336-12.

2 Aquí objetividad no se relaciona con el objetivismo predominante en otras orientaciones investigativas. Por el contrario, remite a un momento metodológico de la investigación: reconstrucción estructural de relaciones sociales.

3 La sociología crítica bourdiana elaborada a lo largo de cuatro décadas de investigaciones realizadas por su autor (Pierre Bourdieu), terminaría siendo expresada de diferentes maneras: Teoría de Campos, Teoría de la Práctica, Praxeología, etc
El otro componente de la perspectiva remitiría a los avances obtenidos en el establecimiento de relaciones de sentido (esquemas y disposiciones) sobre la configuración de dicho "Sistema", los cuales serán expuestos posteriormente, en tanto que la correspondencia entre estructuras objetivas y subjetivas especificaría prácticas y representaciones (Bourdieu, 2013, pp. 13-20), para nuestro caso, prácticas de políticas y significaciones sobre lo público en la educación superior.

Con la investigación se pretende contribuir al incremento comprensivo sobre la posición (menor) ocupada por las universidades públicas dentro del espacio de producción, distribución y concentración de capital escolar en Colombia, entendiendo a éste como expresión de la configuración de un mercado signado por ofertas institucionales y expectativas sociales (probabilidades objetivas) en el acceso a la formación terciaria, cuyas asimetrías constituirían un ordenamiento institucional con prácticas diversificadas y agenciamientos específicos frente a los modos de resolución de sus propios problemas 4 .

\section{Apuesta metodológica}

En la introducción de El oficio de sociólogo, Bourdieu, Passeron y Chamboredon (1975) incorporan un análisis de Comte, relacionándolo con su apuesta de investigación orientada a superar una oposición clásica en la indagación social. Las primeras líneas señalan que:

"El método —escribe Auguste Comte- no es susceptible de ser estudiado separadamente de las investigaciones en que se lo emplea; o, por lo menos, sería éste un estudio muerto, incapaz de fecundar el espíritu que a él se consagre" (Bourdieu, 1975, p. 12).

La pretensión de los autores no se orientaría a refrendar mecánicamente una exposición fechada (la de Comte). Contrariamente, en su teoría del conocimiento de lo social, introducirían dicho planteamiento para invitar a una "vigilancia epistemológica" que, entre otras cosas, debería evitar, en la práctica de investigación, incurrir en la "disociación del método o la teoría respecto de

\footnotetext{
4 En posteriores publicaciones serán desarrollados, además de los componentes indicados, otros más sobre de la orientación teórico metodológica que sustenta la investigación y delineada en el siguiente apartado.
} 
las operaciones de indagación, cuando no disociar la teoría del método o la teoría de la teoría" (p. 12.).

La precitada orientación metodológica asumida para la investigación (Teoría de la práctica), será explicitada en una de sus variantes composicionales, ya que ella misma configura un universo de posibilidades para construir objetos de investigación en donde epistemología, teoría y metodología estarían estrechamente interrelacionadas.

No obstante, previamente se observará que en esta orientación investigativa las prácticas sociales, así como la representaciones sociales, serían resultantes de la interrelación entre estructuras objetivas "independientes de la conciencia y la voluntad" de los agentes sociales y estructuras subjetivas ("esquemas de percepción, apreciación y de acción"), planteamiento conducente a la genérica conceptualización sobre la multicitada noción de campo en diferentes investigaciones:

"[...] configuración de relaciones objetivas entre posiciones [...] [y, en plural] microcosmos sociales relativamente autónomos [...] espacios de relaciones objetivas que son el sitio de una lógica y una necesidad específicas e irreductibles a aquellas que regulan otros campos" (Bourdieu, 2005, pp. 134-135).

Clave comprensiva que indicaría que las posiciones (locus: lugar ocupado) definirían la situación (situs) "presente y potencial" de los agentes, dentro de la estructura de distribución del capital que se juega, además del "[...] acceso a ventajas específicas que están en juego en el campo, así como por su relación objetiva con otras posiciones (dominación, subordinación, homología, etc.)" (2005, pp. 134-135).

Dicho enfoque, allende el "efecto de teoría5", favorecería la desnaturalización de "realidades" sociales, en tanto que superación del denominado sustancialismo, al considerar éste que los fenómenos sociales tendrían existencia y se deberían a sí mismos. La identificación de posiciones dentro del espacio social y los modos de configuración de relaciones, por el contrario, implicarían un trabajo diacrónico y sincrónico con miras

5 Hacer creer que lo que existe en la teoría, existe en la realidad. al establecimiento de su historicidad: "[...] una de las funciones de la teoría de los campos [...] es hacer que la oposición entre reproducción y transformación, lo estático y lo dinámico o estructura e historia se desvanezca". (2005, p. 126). El primero (análisis diacrónico), conduciría a un estudio genésico de la configuración del campo para identificar los referentes de tensión implicados en las posiciones ocupadas por los agentes sociales (Grupos, instituciones).

En el análisis sincrónico, por su parte, se observaría la estructura del campo en un momento determinado y la forma que adquiriría su estructura a partir de las contraposiciones entre los agentes sociales, hallando: "[...] en cada estado sucesivo de la estructura examinada, tanto el producto de luchas previas para mantener o transformar esta estructura, como el principio - a través de las contradicciones, tensiones y relaciones de fuerza que la constituyen- de las subsecuentes transformaciones" (2005, p. 127).

El análisis de la relación entre continuidad y discontinuidad implicaría la identificación precisa de los modos de constitución de los campos, las regularidades instituidas y la emergencia de contraposiciones que darían cuenta de las relaciones de fuerza y de sentido entre los agentes sociales referidas, para el caso que nos ocupa, a la imposición de (re)significaciones políticas.

Breve digresión. Estas breves referencias no apuntan a la clásica definición de categorías que posteriormente serían "identificadas" en la "realidad" social. En El Sentido Práctico (2007), Bourdieu realiza una interesante discusión en términos del Modelo ("Marcos teóricos") y la Ejecución (prácticas sociales, culturales, políticas... de los agentes sociales), en cuya conclusión expresaría que el modelo que está en la cabeza del investigador no está, necesariamente, en la cabeza de los investigados. Si bien sobre el particular habría que realizar otro tipo de publicación, por el momento, queda expresado en el sentido de que la mencionada perspectiva suministra instrumentos conducentes a configurar otra mirada sobre los objetos de investigación en su construcción.

Retomando nuestra discusión previa, se debe anotar que en tanto que nuestro objeto de investigación correspondería al denominado "Sistema" de Educación 


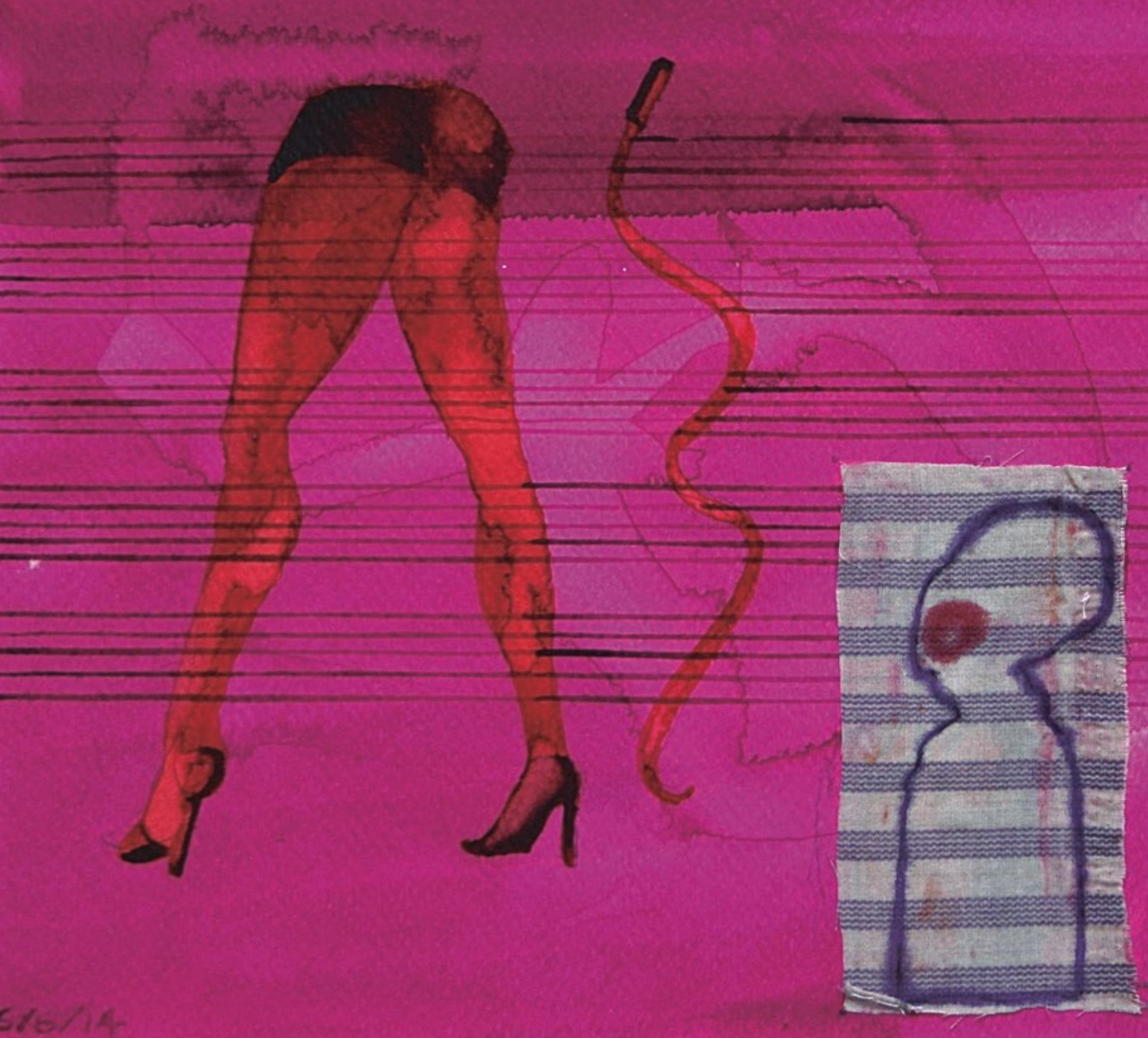

Luis Antonio Sánchez León ॥ Título: Pa' que Afine ॥ Técnica: Mixta » Tamaño: 22 X 21 Cm ॥ Año: 2014 
Superior, la atención quedaría centrada en el período 1992-2012. Consecuentemente, habría necesidad de realizar la debida reconstrucción estadística (diacronía) y análisis documental, cuya expresión quedaría registrada en la tabla y en el gráfico observado en el apartado de resultados.

Estudio que permitiría establecer propiedades constitutivas e inercias subyacentes a las prácticas de políticas entendidas, preliminarmente, como un conjunto de acciones gubernamentales que, si bien se orientan a resolver, entre otros, problemas resultantes de la expansión escolar de tercer nivel, serían consecuencia de los agenciamientos interesados (grupos e instituciones) dentro de su configuración histórica.

La investigación estuvo signada por la relectura de investigaciones especializadas, principalmente la de Ricardo Lucio y Mariana Serrano (1992), la proyección de trabajos realizados (Guevara, 2009, 2012), y la construcción de datos a partir de información estadística publicada en su momento por el ICFES y la reportada al Sistema Nacional de Información de la Educación Superior (SNIES), sobre dos variables (Instituciones y Matrícula de educación superior) que favorecerían el trazado de un marco temporal reconstructivo (1935-2012).

Este trabajo permitirá observar la relación entre oferta institucional pública y oferta institucional privada, para comprender cómo se configuraría la competencia sectorial público-privado: un estado de cosas entre instituciones que contenderían frente a las posibilidades de acceso a la educación superior colombiana, en donde solo el $11 \%$ del total de Instituciones de Educación Superior (IES), en 2012, son universidades públicas.

Finalmente, algunos pasajes de los resultados introducen datos sobre la participación diferencial institucional (Técnicas, tecnológicas...) y la distribución por áreas del conocimiento con miras a perfilar análisis que serán compartidos posteriormente.

\section{Resultados}

\section{Luchas partidistas por acceder al poder del Estado: síntesis histórica}

El siglo XX colombiano heredaría del siglo XIX un acumulado de confrontaciones, alternancias y rediseños institucionales en el acceso al dominio partidista de los bienes públicos concentrados en el Estado, en tanto que partícipes de las condiciones de posibilidad que delimitarían los procesos que configurarían políticas en educación superior (Lucio y Serrano, 1992).

La Guerra de los Mil Días (1899-1902), entre liberales y conservadores, proyectaría antagonismos políticos marcados por la violencia partidista decimonónica. Hasta 1930, los presidentes provendrían del Partido Conservador. Entre 1930 y 1946 vendría una sucesión de presidentes Liberales, seguida de un nuevo período de presidentes Conservadores (1946-1953), interrumpido por la Dictadura Militar (1953-1958) cuyo principal exponente renunciaría para dar paso al Frente Nacional (1958-1974): acuerdo de alternancias entre liberales y conservadores.

Entre 1974 y 1990 habría tres presidentes liberales (1974-1978-1982 y 1986-1990) y uno conservador (1986-1990). Progresivamente el conflicto liberalconservador sería atenuado por otras confrontaciones (Guerrilla, violencia del narcotráfico) que fungirían condiciones de posibilidad para el reordenamiento constitucional de 1991. En adelante, la reconfiguración política nacional, la desmovilización-reinserción de grupos armados y la apertura del espectro electoral a otros ordenamientos políticos, darían paso a presidentes formados en los partidos tradicionales, quienes serían electos por opciones partidistas que desdibujarían los límites característicos de las contiendas precedentes.

La nueva constitución (1991), sería resultado de una Asamblea Constituyente convocada en un gobierno liberal (1990-1994) seguido de otro del mismo partido (1994-1998). Entre 1998 y 2002 retornarían los conservadores. En 2002 sería elegido presidente el candidato 
de una coalición política proveniente de los partidos históricos (liberal-conservador), reelegido (2006-2010) y, para el período siguiente (2010-2014), sería electo un nuevo candidato del mismo partido.

Finalmente, los años que van, aproximadamente, de finales de la segunda guerra mundial (1945), hasta comienzos de la década de los noventa del siglo XX, estarían caracterizados por la sustitución de importaciones (modelo de desarrollo). La transformación constitucional de 1991 sería la ocasión propicia para instituir un nuevo modelo amparado en la apertura económica, con implicaciones sobre las políticas sectoriales, entre otras, las de educación superior.

Esta breve descripción contribuye a comprender los elementos que serán resituados a continuación.

\section{Institucionalización del predominio privado en la educación superior colombiana}

En el espacio de la educación superior colombiana se observa que, durante los gobiernos liberales (1930-1946), las Instituciones de Educación Superior (IES), herederas republicanismo decimonónico, ascenderían a doce (12), con una participación relativa de instituciones estatales, públicas u oficiales (denominaciones sinónimas) del $67 \%$ y una matrícula del $71 \%$, mientras que su contraparte, las instituciones privadas (No oficiales), serían el restante 33\% con una matrícula del 29\%. (Tabla 1.).

Al finalizar la República Liberal (1946), las IES aumentarían a diecinueve (19), seis de las cuales serían nuevas en el sector público, expresando un aumento cercano al 75\%, mientras que el sector no oficial o privado aumentaría en una IES. La participación pública en oferta institucional y matrícula ascenderían al 74\%. La oferta institucional y matrícula privadas serían del $26 \%$.

Tabla 1. Colombia. Distribución sectorial de IES y de la Matrícula. 1823-2012

\begin{tabular}{|c|c|c|c|c|c|c|c|c|c|}
\hline \multirow[b]{2}{*}{ Año } & \multicolumn{2}{|c|}{ Institución } & \multicolumn{2}{|c|}{ Matrícula } & \multirow[b]{2}{*}{ Año } & \multicolumn{2}{|c|}{ Institución } & \multicolumn{2}{|c|}{ Matrícula } \\
\hline & Oficial & Privada & Oficial & Privada & & Oficial & Privada & Oficial & Privada \\
\hline 1823 & 50 & 50 & ND & ND & 1971 & 42 & 58 & 52 & 48 \\
\hline 1827 & 63 & 38 & ND & ND & 1972 & 42 & 58 & 53 & 47 \\
\hline 1867 & 86 & 14 & ND & ND & 1973 & 40 & 60 & 50 & 50 \\
\hline 1886 & 75 & 25 & ND & ND & 1974 & 39 & 61 & 49 & 51 \\
\hline 1903 & 78 & 22 & ND & ND & 1975 & 41 & 59 & 49 & 51 \\
\hline 1923 & 70 & 30 & ND & ND & 1976 & 41 & 59 & 49 & 51 \\
\hline 1931 & 67 & 33 & ND & ND & 1977 & 39 & 61 & 50 & 50 \\
\hline 1935 & 67 & 33 & 71 & 29 & 1978 & 40 & 60 & 48 & 52 \\
\hline 1936 & 64 & 36 & 69 & 28 & 1979 & 40 & 60 & 45 & 55 \\
\hline 1937 & 64 & 36 & 72 & 28 & 1980 & 30 & 70 & 37 & 63 \\
\hline 1938 & 64 & 36 & 78 & 22 & 1981 & 30 & 71 & 40 & 60 \\
\hline 1939 & 64 & 36 & 77 & 23 & 1982 & 30 & 70 & 39 & 61 \\
\hline 1940 & 64 & 36 & 68 & 32 & 1983 & 30 & 70 & 40 & 60 \\
\hline 1941 & 67 & 33 & 71 & 29 & 1984 & 30 & 70 & 39 & 61 \\
\hline 1942 & 67 & 33 & 74 & 26 & 1985 & 31 & 69 & 40 & 60 \\
\hline 1943 & 69 & 31 & 72 & 28 & 1986 & 31 & 69 & 42 & 58 \\
\hline 1944 & 71 & 29 & 72 & 28 & 1987 & 30 & 70 & 42 & 58 \\
\hline 1945 & 74 & 26 & 73 & 27 & 1988 & 29 & 71 & 41 & 59 \\
\hline 1946 & 74 & 26 & 74 & 26 & 1989 & 29 & 71 & 41 & 59 \\
\hline 1947 & 74 & 26 & 74 & 26 & 1990 & 31 & 69 & 40 & 60 \\
\hline 1948 & 73 & 27 & 75 & 25 & 1991 & 30 & 70 & 39 & 61 \\
\hline 1949 & 73 & 27 & 74 & 26 & 1992 & 30 & 70 & 36 & 64 \\
\hline 1950 & 70 & 30 & 72 & 28 & 1993 & 30 & 70 & 35 & 65 \\
\hline
\end{tabular}




\begin{tabular}{llllllllll}
1951 & 67 & 33 & 68 & 32 & 1994 & 31 & 69 & 35 & 65 \\
1952 & 64 & 36 & 67 & 33 & 1995 & 30 & 70 & 33 & 67 \\
1953 & 64 & 36 & 66 & 34 & 1996 & 30 & 70 & 32 & 68 \\
1954 & 59 & 41 & 65 & 35 & 1997 & 30 & 70 & 33 & 67 \\
1955 & 59 & 41 & 62 & 38 & 1998 & 31 & 69 & 35 & 65 \\
1956 & 59 & 41 & 61 & 39 & 1999 & 31 & 69 & 34 & 66 \\
1957 & 59 & 41 & 60 & 40 & 2000 & 32 & 68 & 37 & 64 \\
1958 & 57 & 43 & 58 & 42 & 2001 & 32 & 68 & 38 & 62 \\
1959 & 57 & 43 & 59 & 41 & 2002 & 32 & 68 & 42 & 58 \\
1960 & 55 & 45 & 59 & 41 & 2003 & 29 & 71 & 45 & 55 \\
\hline 1961 & 58 & 42 & 58 & 42 & 2004 & 29 & 71 & 49 & 51 \\
1962 & 62 & 38 & 57 & 43 & 2005 & 29 & 71 & 49 & 51 \\
1963 & 62 & 38 & 57 & 43 & 2006 & 29 & 71 & 51 & 49 \\
\hline 1964 & 63 & 37 & 58 & 42 & 2007 & 29 & 71 & 54 & 46 \\
\hline 1965 & 59 & 41 & 57 & 43 & 2008 & 29 & 71 & 55 & 45 \\
\hline 1966 & 58 & 43 & 57 & 43 & 2009 & 28 & 72 & 56 & 44 \\
\hline 1967 & 58 & 43 & 55 & 45 & 2010 & 28 & 72 & 55 & 45 \\
\hline 1968 & 58 & 43 & 55 & 45 & 2011 & 28 & 72 & 54 & 46 \\
\hline 1969 & 58 & 43 & 54 & 46 & 2012 & 28 & 72 & 53 & 47 \\
\hline 1970 & 49 & 51 & 54 & 46 & & & & & \\
\hline & & & & & & & & & \\
\hline 1969
\end{tabular}

Brevemente, a pesar del bajo número de IES, dadas las condiciones históricas, políticas, económicas y sociales de la época, la finalización de la República Liberal ubicaría una posición irrepetible en la institucionalidad pública de la educación superior colombiana: casi las tres cuartas partes de las IES y de la matrícula serían públicas, oficiales o estatales, tal y como se observa en la siguiente expresión gráfica de los datos precedentes.

Grafico 1. Institucionalización del predominio privado en la educación superior en Colombia

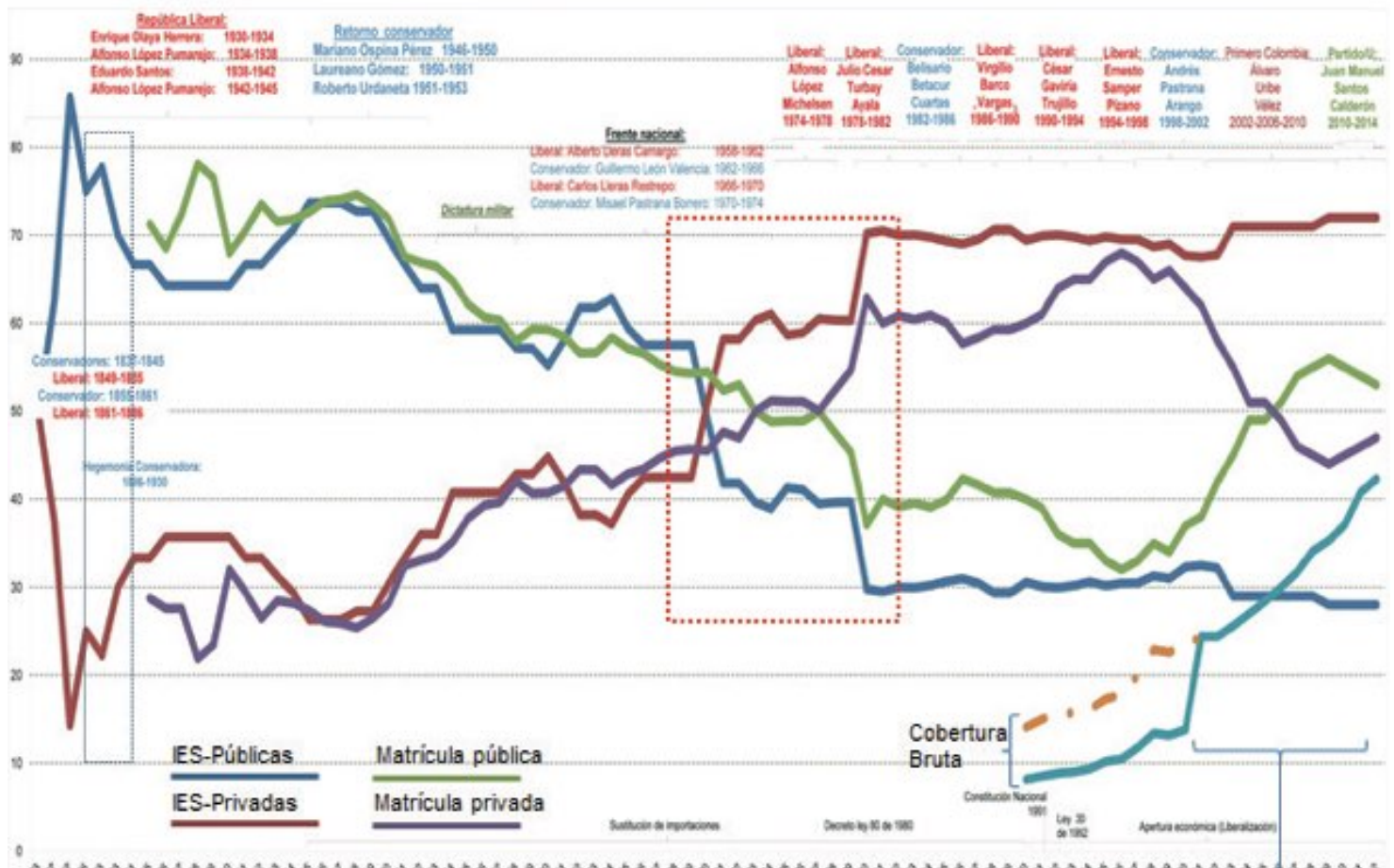


Los gobiernos conservadores (1946-1953), arrojarían como "resultado" que del total de IES para ese momento (25), las públicas habrían aumentado su presencia relativa en solo $14 \%$ (dos nuevas IES), mientras que las privadas lo harían en $80 \%$ (cinco nuevas IES). De esta manera, la oferta institucional pública vería reducida su proporcionalidad frente a la expresada en 1935. En este año las IES públicas serían el $67 \%$, con una matrícula del $71 \%$. Las IES privadas serían el 33\% con una matrícula del 29\%. En 1953, las IES públicas disminuirían al $64 \%$ con $66 \%$ de matrícula. Las IES privadas corresponderían al 36\% y una matrícula del 34\%.

Este dato es clave en la indagación, toda vez que marca una inflexión política irreversible en la presencia estatal dentro de la oferta institucional y en la matrícula de educación superior. Si bien desde la fundación de las universidades republicanas, a finales del siglo XIX, las universidades públicas tendrían una fuerte presencia afianzada con las políticas de López Pumajero (19351938), con el retorno de los conservadores al gobierno nacional, la tendencia se agudizaría al punto de tornarse contraria, con un predominio institucional privado y una fuerte competencia en la matrícula de educación con períodos de alternancia público-privado.

Efectivamente, la Dictadura Militar (1953-1958)6 significaría un estancamiento de la oferta institucional pública y la consolidación de los acumulados del sector privado. Las primeras continuarían con el mismo número de IES de 1951 (16), mientras las IES privadas aumentarían 33\%, al pasar de nueve IES en 1953 a doce IES en 1958. Las cuentas darían que, a la finalización de la Dictadura (1958), la situación sería muy próxima al equilibrio tanto en el plano institucional como en la matrícula: IES públicas con $57 \%$ de oferta institucional

6 Se debe anotar que comenzando este momento de la historia colombiana sería creado el Fondo Universitario Nacional -FUN (1954), con el fin de proveer el desarrollo de las universidades colombianas según lo expresara el Decreto 3686 de 1954. A su vez, en la extinción de la dictadura sería creada la Asociación Colombiana de Universidades, ASCUN (1957). En 1958 serían fusionadas y reconocidas como ASCUN-FUN. En 1968 sería disuelta la "sociedad" debido a la creación del Instituto Colombiano para el Fomento de la Educación Superior, ICFES, en medio de la reforma del Estado de Carlos Lleras Restrepo. Estos aspectos serán desarrollados en otro artículo en elaboración a propósito de la autonomía política universitaria, en correspondencia con los antagonismos expresados en el presente artículo. y $58 \%$ de matrícula, y IES privadas con $43 \%$ de oferta institucional y $42 \%$ en matrícula.

El Frente Nacional (1958-1974) consagraría lo descrito sin que sea leído como parte del referido pacto político. Los datos observados serían más consecuencia de dejar en manos del mercado la configuración del espacio de la educación superior, que de una acción deliberada por parte de los gobernantes de esos años, cuya principal función constitucional sería salvaguardada: inspección y vigilancia. También se crearían varias agencias públicas: ASCUN, ICFES, ICETEX.

Las IES se incrementarían en casi tres veces y media al pasar de veintiocho en 1958 a noventa y cinco en 1974: aumento del $340 \%$. De manera desagregada, las IES públicas aumentarían poco más del doble (130\%), mientras las privadas lo harían en más del 380\%. Afirmación de la regularidad reproducida en las décadas por venir, ya que la oferta institucional pública sería del 39\% con matrícula del $49 \%$. El sector privado correspondería al $61 \%$ de oferta institucional y $51 \%$ de matrícula.

Siendo rigurosos con las fechas, se puede afirmar que en $1970^{7}$ se instala el predominio del sector privado (51\%) en la oferta institucional de la educación superior en Colombia, contribuyendo a que, posteriormente, durante el primer gobierno Pos-Frente Nacional (19741978), en 1974, la matrícula de la educación superior replicara dicha experiencia concentrando la participación del sector privado con un 51\%.

La situación de 1946, un poco más de tres décadas después (1978), sería revertida en medio de varios procesos característicos del país de entonces: mesocratización, urbanización, expansión demográfica, concentración poblacional en los principales centros urbanos. Situación que instituiría más que un "sistema mixto" de educación superior, como indicarían algunos comentaristas, el predominio institucional del sector privado y la alternancia en la matrícula de la educación superior colombiana.

\footnotetext{
7 En el gráfico, el recuadro rojo punteado señala el fenómeno estadístico identificado.
} 
Incluyendo, de aquí en adelante, la matrícula por áreas del conocimiento8, tenemos que en este mismo período (1958-1974) poco más de las tres cuartas partes, en 1960, se ubicaría en Artes (11\%), ciencias de la salud y derecho y ciencias sociales (cada una con $21 \%$ ) e ingenierías (24\%). Hacia 1974, la matrícula por áreas vería un desplazamiento del primer lugar, al ser encabezada por los programas de Administración, Economía y afines (26\%), seguidos de Ingeniería (22\%) y Educación y Derecho (ambos con 17\% cada uno).

Ante los cuestionamientos a la situación acumulada en la educación superior, en 1980 el Presidente de la República, en cumplimiento de facultades extraordinarias otorgadas por el Congreso (Ley $8^{a}$ de 1979), expediría el Decreto-ley 80 de 1980, con miras a poner en orden la denominada Educación Post-secundaria, regulada hasta ese entonces por decretos dispersos; dicha normativa dispondría tres ámbitos generales de regulación sectorial:

Sistema de Educación Post-secundaria: naturaleza del sistema; unificar regímenes y programas; armonizar relaciones entre IES y autoridades de su orientación, vigilancia y funcionamiento.

Institucional: fijar requisitos y procedimientos para su creación y funcionamiento, según planes de desarrollo; democratizar y descentralizar la enseñanza universitaria y tecnológica.

Fijar requisitos tarifarios para matrículas.

En cuanto a las IES públicas, específicamente, además de reorganizar la Universidad Nacional de Colombia, señalaría la necesaria realización de una situación similar para el conjunto de IES oficiales, en cuanto a sus estatutos orgánicos, definiendo: naturaleza jurídica, órganos de dirección -composición y funciones-, administración, calidades y atribuciones de directivos, sujeción a las normas administrativas y financieras de planificación en tanto que establecimientos públicos, regímenes de personal y estudiantil.

8 En otro momento será complementada la retrospectiva de estos datos, ya que la información recopilada sobre el particular carece de la suficiente consistencia, tal y como lo reseñan, entre otros especialistas, Lucio y Serrano (1992).
Si el Decreto 80 de 1980 señalaba que en adelante el reconocimiento de universidad implicaría poseer mínimo tres programas profesionales (Art. 47), se puede inferir que un porcentaje de IES universitarias lo serían con pocos programas profesionales y varios de orden Técnico-Profesional o Tecnológico. El panorama de la oferta institucional especifica un total de ciento ochenta y ocho IES: $30 \%$ públicas, con $37 \%$ de la matrícula, y $70 \%$ privadas con el $63 \%$ de la matrícula.

Incorporando al análisis el carácter institucional, a partir de este momento, según clasificación oficial de la época, tendríamos: Instituciones Universitarias, 58\%; Instituciones Tecnológicas, 11\%; Instituciones Técnico Profesionales o Intermedias Profesionales, 31\%. EI panorama diferenciado por sectores expresa que la oferta pública sería minoritaria en Técnico Profesional (3\%), aumentaría en Instituciones Tecnológicas (40\%) e Instituciones Universitarias (42\%). El predominio del sector privado sería pleno: el 97\% en Técnico Profesional, $60 \%$ en Instituciones Tecnológicas y $58 \%$ en Instituciones Universitarias.

La matrícula por Áreas del Conocimiento conservaría la situación mencionada anteriormente, salvo el aumento en Administración, Economía y afines (32\%), seguida de ingeniería (18\%), Educación (16\%) y Derecho (15\%). Áreas que concentrarían el $82 \%$ de matrícula en educación superior.

En tanto que campo o "red de relaciones objetivas... que ejercen mutuos efectos a distancia" (Bourdieu, 2013, p. 189), los datos observados evidenciarían que, concomitante con los antagonismos políticos referidos previamente, la posible ausencia de un proyecto público sobre la universidad aparejaría un incremento del sector privado, en donde las IES públicas terminarían ocupando un lugar subordinado a pesar de su importancia social.

Brevemente, los acumulados públicos en educación superior, obtenidos durante los gobiernos liberales (1930-1946), disminuirían progresivamente durante los gobiernos conservadores (1946-1953). La Dictadura (1953-1958) los agudizaría y, durante el Frente Nacional (1958-1974), se consolidaría el predominio privado. Con el decreto 80/80 se intentaría poner orden a la expansión sin planificación, constituyendo un 
primer momento en la configuración de un campo de políticas para la educación superior, centrado en tres temas: cobertura, calidad y eficiencia.

Una década después (1991), con doscientos cuarenta y seis IES, el incremento sería similar en cada sector, frente a 1980: 130\% c/u. La diferenciación institucional por carácter reafirmaría el predominio privado en la educación superior: si bien las Instituciones TécnicoProfesionales públicas aumentarían a $17 \%$, el sector privado concentraría la mayor presencia con $83 \%$. En Instituciones Tecnológicas, por el contrario, mientras el sector privado incrementaría su presencia a $67 \%$, el sector público lo perdería disminuyendo al 33\%.

La ley de educación superior (No 30 de 1992) diferenció entre Instituciones Universitarias (Formación profesional) y universidades responsables, además, de la generación de conocimiento. La diferenciación sectorial sería del $72 \%$ para las primeras y $59 \%$ para las segundas en el sector privado. La contraparte, sector público, tendría 28\% para Instituciones Universitarias y $41 \%$ para Universidades.

La matrícula pública (1992) sería del 30\%, frente a un $70 \%$ del sector privado. Por carácter institucional, las universidades concentrarían el 30\%, las Instituciones Universitarias el 26\%, las Instituciones Tecnológicas el $20 \%$ y las Instituciones Técnico Profesionales el 24\%. Las IES públicas tendrían participación minoritaria: $17 \%$ en Instituciones Técnico Profesionales, 33\% en Instituciones Tecnológicas, 28\% en Instituciones Universitarias y $41 \%$ en Universidades.

Aunque se avanzaría en la reordenación institucional pretendida (Decreto 80/1980), también se reafirmaría el predominio privado. La acción gubernamental confirmaría la "suprema inspección y vigilancia" en la regulación de la oferta institucional. Las IES privadas corresponderían al $70 \%$ y una matrícula del $64 \%$. Las IES públicas $30 \%$ y matrícula del $36 \%$. La tasa de cobertura en educación superior (Tabla 2) sería del 9\%9 .

9 El gráfico presentado incorpora datos relacionados con la cobertura bruta a partir de 1990, diferenciados con líneas de dos colores: naranja, correspondiente a reconstrucción de datos históricos del ICFES; con la creación y puesta en funcionamiento del Viceministerio de Educación Superior 2002-2003, estas estadísticas están siendo reformuladas a partir del nuevo rango etario definido para calcular la cobertura bruta.
Por Áreas del Conocimiento: 73\% concentrada en Administración, Economía y afines (29\%), Ingenierías (26\%) y conservando la distancia frente a Educación (16\%) y Derecho (12\%).

Tabla 2. Tasa de Cobertura

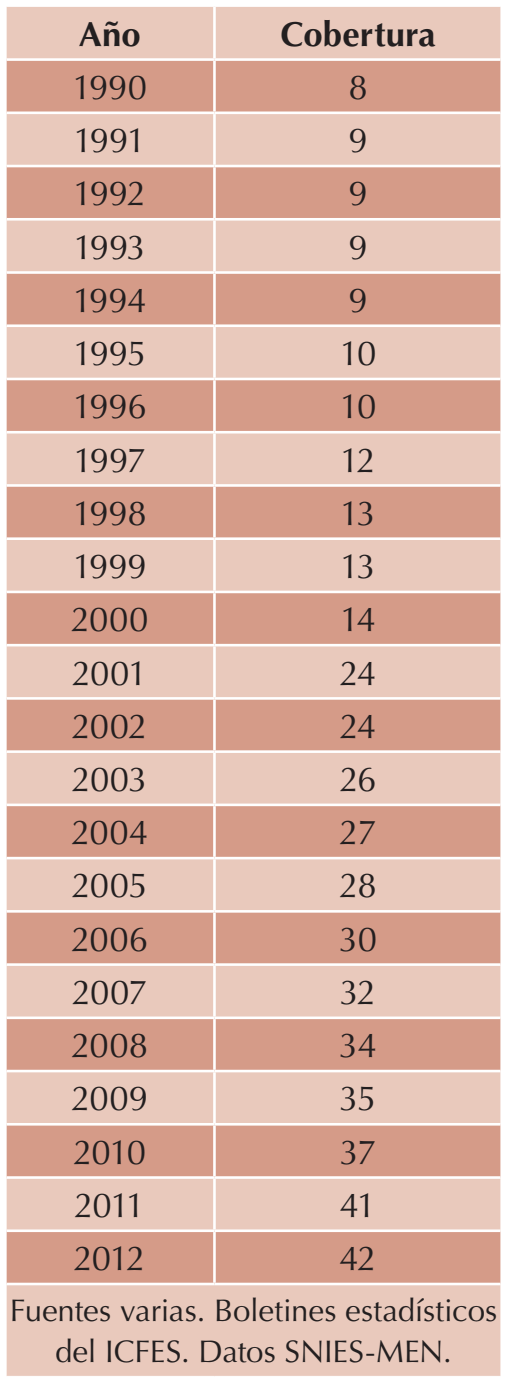

Ahora bien, los años 1992-2012 se diferenciarían del período 1992-2002, al ser dispuesto un conjunto de procesos, dinámicas y eventos orientados a la puesta en práctica de la Ley 30 de 1992. La segunda (20022012), consolidaría el campo a partir de un conjunto de políticas gubernamentales implementadas como consecuencia de los acumulados previos. 
En la primera década (1992-2002), a diferencia de los años sesenta y setenta, un importante "contingente" de académicos universitarios ${ }^{10}$ participaría en la elaboración de diagnósticos y recomendaciones mediante las Ilamadas Misiones (Ciencia, Educación y Desarrollo o "Misión de Sabios": 1993-1995; Modernización de la Universidad Pública: 1994-1997; Comisión Nacional para el Desarrollo de la Educación Superior: 1996), precedidas de la Misión de Ciencia y Tecnología (1988). Época en donde las universidades públicas se esforzarían por realizar la autonomía académica (Reforma curricular), posteriormente soslayada por la institucionalización de los procesos de acreditación, la extensión de la política de competencias hacia la educación superior y el establecimiento de los Registros Calificados (CONACES).

Los datos reafirmarían la presencia institucional privada con $68 \%$ y una matrícula del $58 \%$. Las IES públicas corresponderían al 32\% con matrícula del $42 \%$. La concentración estaría en programas de Ingeniería (30\%), seguidos cercanamente por Administración, Economía y afines (28\%), Derecho (14\%) y Salud (11\%).

La cobertura ascendería al 24\% con una anotación. En 2000 correspondería a 14\%; en 2001 ascendería al 24\% y en 2012 llegaría al 42\%. Variación comprensible por varias acciones de políticas: la reducción del rango etario internacional (18-24 años) a 17-21 años durante 2000-2002; la transformación del Instituto Colombiano para el Fomento a la Educación Superior (ICFES) en Instituto de Evaluación, y el traslado de su responsabilidad estadística al Viceministerio de Educación; la incorporación estadística de la matrícula estudiantil del Servicio Nacional de Aprendizaje (SENA), y el incremento de nuevos cupos en las universidades públicas ${ }^{11}$.

\footnotetext{
10 En las décadas señaladas, las misiones extranjeras asesoraban los procesos de reforma de la educación superior colombiana. Por ejemplo, la participación de académicos de la Universidad de California en la formulación del denominado Plan Básico de la Educación Superior de 1966. Experiencia que sería reinstituida en 2012 con la asesoría expresa de la OCDE-Banco Mundial.
}

11 Este análisis será desarrollado en otro artículo en elaboración, a propósito de las variaciones de la cobertura en la educación superior durante los dos gobiernos de Uribe Vélez y la participación de las universidades públicas a partir de la consolidación del Sistema Universitario Estatal (SUE).
La distribución por sectores daría un saldo a favor del sector público (21\%) en Instituciones Técnico-Profesionales frente a las privadas (79\%). En Instituciones Tecnológicas sería similar a las observadas previamente (privadas: 68\%-públicas: 32\%). Igual ocurriría en Instituciones Universitarias (privadas: 26\%-públicas: $74 \%$ ). A nivel universitario: sector público, 44\%; sector privado, 56\%.

El último momento de políticas (2002-2012) correspondería a la institucionalización del Sistema de Aseguramiento de la Calidad de la Educación Superior (SACES); el fortalecimiento del SNIES y la puesta en funcionamiento de los Sistemas de Prevención de la Deserción de la Educación Superior (SPADIES), y de Evaluación Docente de la Educación Superior (SNEDES). También, la creación del Observatorio Laboral.

Por primera vez, en décadas, se dispondría de bases de datos orientadas a la construcción de información asociada a la toma de decisiones de políticas, que ofrecerían la oportunidad para indagar sobre desempeños institucionales e implementación de políticas para la transformación de la educación superior. Hacia 2012 la oferta de IES no presenta variaciones: $28 \%$ pública y $72 \%$ privada. La novedad se encontraría en la matrícula, ya que, a diferencia de 2002, el sector público repuntaría al 53\%. La matrícula privada se contraería al 47\%12.

12 Como fue anotado previamente, este repunte del sector público obedecería a la participación de las universidades públicas, que incrementaron significativamente la matrícula; la incorporación del SENA con sus programas técnicos y tecnológicos; y la variación del rango etario para calcular la tasa de cobertura. 


\begin{tabular}{|c|c|c|c|c|c|c|c|c|c|c|c|c|c|c|}
\hline \multirow[b]{4}{*}{ Carácter } & \multicolumn{14}{|c|}{$\begin{array}{l}\text { Tabla 3. Instituciones de educación superior principales. Instituciones de educación superior. } \\
\text { Distribución y participación por sector y carácter académico. 2010, 2012. (Sincronía política) }\end{array}$} \\
\hline & \multirow{2}{*}{\multicolumn{2}{|c|}{ Total }} & \multicolumn{6}{|c|}{2010} & \multicolumn{6}{|c|}{2012} \\
\hline & & & \multicolumn{2}{|c|}{ Total IES } & \multicolumn{2}{|c|}{$\begin{array}{l}\text { Dentro del } \\
\text { propio carácter }\end{array}$} & \multicolumn{2}{|c|}{$\begin{array}{l}\text { Dentro del } \\
\text { propio sector }\end{array}$} & \multicolumn{2}{|c|}{ Total IES } & \multicolumn{2}{|c|}{$\begin{array}{l}\text { Dentro del } \\
\text { propio carácter }\end{array}$} & \multicolumn{2}{|c|}{$\begin{array}{l}\text { Dentro del } \\
\text { propio sector }\end{array}$} \\
\hline & 2010 & 2012 & Oficial & Privado & Oficial & Privado & Oficial & Privado & Oficial & Privado & Oficial & Privado & Oficial & Privado \\
\hline Universidad & 80 & 81 & 11 & 17 & 40 & 60 & 40 & 23 & 11 & 17 & 39 & 60 & 51 & 24 \\
\hline $\begin{array}{l}\text { Institución universitaria/ } \\
\text { Escuela tecnológica }\end{array}$ & 114 & 120 & 9 & 30 & 24 & 76 & 34 & 42 & 9 & 32 & 23 & 77 & 25 & 45 \\
\hline Institución tecnológica & 51 & 50 & 4 & 14 & 24 & 76 & 15 & 19 & 4 & 13 & 24 & 76 & 10 & 18 \\
\hline $\begin{array}{l}\text { Institución técnica } \\
\text { profesional }\end{array}$ & 41 & 37 & 3 & 11 & 22 & 78 & 11 & 16 & 3 & 10 & 24 & 76 & 15 & 13 \\
\hline Total general & 286 & 288 & 28 & 72 & & & 100 & 100 & 28 & 72 & & & 100 & 100 \\
\hline Valores & \multicolumn{2}{|c|}{ Absolutos } & \multicolumn{12}{|c|}{ Relativos } \\
\hline
\end{tabular}

En la Tabla 3 se observa que las IES reconocidas como universidades (2012) serían 81. Las universidades públicas corresponderían al $11 \%$, mientras que las universidades privadas serían el $17 \%$ del total de 288 IES. Dentro de su universo particular (universidades), el $40 \%$ serían públicas y el $60 \%$ privadas. En su sector específico, las universidades públicas corresponderían casi al $40 \%$ de IES públicas. Las privadas serían el $24 \%$ dentro del conjunto de IES privadas.

\section{Conclusiones}

La educación superior colombiana contendría un predominio privado, históricamente situado, fechado e identificado institucionalmente hacia 1970, y en matrícula en 1974; luego, a partir de la década de 1970, las universidades públicas ocuparían un lugar subordinado con todo y que las exigencias de políticas se circunscribirían, en la justificación de su implementación, a este subsector.

Los argumentos conducentes a la expedición del Decreto 80 de 1980 y, posteriormente, la ley que sería numerada como 30 de 1992, tendrían como justificación "poner en orden" el "caos" institucional observado, ya que el incremento institucional de la educación superior aparejaría problemas de cobertura, calidad y eficiencia.
No obstante, se podría interrogar por las consecuencias sobre el desempeño de las IES privadas, en el devenir instituido, a propósito de la implementación de políticas de evaluación de la calidad de la educación superior (programas académicos e instituciones): ¿cuántos programas académicos e instituciones de nivel técnico, tecnológico y profesional de carácter privado han sido objeto de cierre, reformulación o condicionamiento como consecuencia de su participación en los procesos de evaluación de la calidad? Aún más: ¿cuál es su nivel de participación en dichos procesos, toda vez que se caracteriza por la voluntariedad?

La información estadística recopilada y el análisis documental realizado, cuya expresión está contenida en las tablas y en el gráfico incorporados previamente, permitiría identificar tres momentos institucionales en la oposición público-privado: el primero hasta 1946, caracterizado por una fuerte presencia del sector público en los dos planos analíticos, instituciones y matrícula. El segundo, centrado en la década de 1970, en donde, a pesar del leve repunte institucional en los gobiernos liberales (1958-1962 y 1966-1970), se consolidaría el predominio institucional del sector privado. La última, a partir de 1980 con el afianzamiento institucional del sector privado frente al público y una alternancia en la participación de matrícula consolidada, con el repunte observado identificable durante el gobierno de Uribe Vélez y las debidas consecuencias sobre la política de financiación para las universidades públicas. 
Esta primera incursión, amparada en la perspectiva de investigación que continuará siendo robustecida, salvaguarda el objetivo anotado: participar en la desnaturalización de los procesos de políticas en la educación superior colombiana. Posteriormente, habrá ocasión de profundizar sobre otros asuntos analíticos (distribución público-privado de: jornadas académicas, oferta de programas académicos, recursos operacionales, planta administrativa y docente, financiación y fuentes

\section{Referencias}

Bourdieu, P. (1975). El oficio de sociólogo. Buenos Aires: Siglo XXI editores.

Bourdieu, P. (2007). El sentido práctico. Buenos Aires: Siglo $\mathrm{XXI}$ editores.

Bourdieu, P. (2013). La nobleza de Estado. Buenos Aires: Siglo $\mathrm{XXI}$ editores.

Bourdieu, P., y Wacquant, L. (2005). Una invitación a la sociología reflexiva. Buenos Aires: Siglo XXI editores.

Lucio, R., y Serrano, M. (1992). La Educación Superior. Tendencias y Políticas Estatales. Instituto de Estudios Políticos y Relaciones Internacionales, Universidad Nacional de Colombia. Bogotá: Tercer Mundo Editores.

Guevara, R. (2009). Universidades públicas, autonomía política universitaria y elección de rectores: prefiguración del Campo Político Universitario. Revista de Pedagogía y Saberes, 31. Universidad Pedagógica Nacional. Colombia.

Guevara, R. (2012). Nominación rectoral y colegios electorales universitarios. Estudio de casos: UAM (México), UPN, (Colombia). Tesis doctoral. México: Universidad Autónoma Metropolitana. Unidad Xochimilco. presupuestales, matrícula por niveles institucionales y áreas del conocimiento), así como de exponer los mecanismos a través de los cuales el nomos de políticas, la educación como servicio público, sería instituido en tanto que el mismo determinaría la comprensión que se reproduce sobre la educación superior colombiana dentro de los principios de visión y de división de quienes toman decisiones sectoriales.

\section{Fuentes oficiales}

Instituto colombiano para el Fomento de la Educación Superior, ICFES. Boletines estadísticos.

Ministerio de Educación Nacional. www.mineducacion.gov. co Sistema Nacional de Información de la Educación Superior. SNIES. 